\title{
Berberine prevents the apoptosis of mouse podocytes induced by TRAF5 overexpression by suppressing NF-кB activation
}

\author{
FENG WU ${ }^{1,2}$, DONG-SHENG YAO ${ }^{1,2}$, TIAN-YING LAN ${ }^{1,2}$, CHEN WANG $^{1,2}$, \\ JIAN-DONG GAO ${ }^{2,3}$, LI-QUN HE ${ }^{1,2}$ and DI HUANG ${ }^{2,3}$ \\ ${ }^{1}$ Department of Nephrology, Shuguang Hospital of Shanghai University of Traditional Chinese Medicine; \\ ${ }^{2}$ Shanghai Key Laboratory of Traditional Chinese Clinical Medicine; ${ }^{3}$ TCM Institute of Kidney Disease, \\ Shanghai University of Traditional Chinese Medicine, Shanghai 201203, P.R. China
}

Received June 14, 2016; Accepted September 28, 2017

DOI: $10.3892 /$ ijmm.2017.3236

\begin{abstract}
Berberine (BBR) has previously been found to exert beneficial effects on renal injury in experimental rats. However, the mechanisms underlying these effects are not yet fully understood. Tumor necrosis factor (TNF) receptor-associated factor 5 (TRAF5) has been demonstrated to mediate the activation of nuclear factor $-\kappa \mathrm{B}(\mathrm{NF}-\kappa \mathrm{B})$, which has been implicated in the pathogenesis of chronic kidney disease (CKD). The aim of this study was to investigate the effects of BBR on kidney injury and the activation of the $\mathrm{NF}-\kappa \mathrm{B}$ signaling pathway in mouse podocytes. TRAF5 was found to be overexpressed in patients with CKD and chronic renal failure (CRF) (data obtained from the dataset GSE48944, as well as from patients at Shuguang Hospital). TRAF5 overexpression significantly inhibited cell viability and induced the apoptosis of mouse podocytes. However, BBR prevented the decrease in cell viability and the apoptosis induced by TRAF5 overexpression. The NF- $\mathrm{B}$ inhibitor, caffeic acid phenethyl ester (CAPE), mimicked the protective effects of $\mathrm{BBR}$, as evidenced by the increased expression of nephrin and podocin, and the decreased the expression of caspase- 3 and the ratio of $\mathrm{Bax} / \mathrm{Bcl}-2$. Moreover, BBR prevented the decrease in cell viability decrease and the apoptosis induced by TNF- $\alpha$, interleukin (IL)- 6 and lipopolysaccharide (LPS). Taken together, our data indicate that BBR exerts protective effects against CRF partly through the TRAF5-mediated activation of the NF- $\kappa \mathrm{B}$ signaling pathway in mouse podocytes.
\end{abstract}

\section{Introduction}

Chronic renal failure (CRF) is considered the most severe outcome of chronic kidney disease (CKD) and is defined

Correspondence to: Dr Di Huang, TCM Institute of Kidney Disease, Shanghai University of Traditional Chinese Medicine, 528 Zhang Heng Road, Shanghai 201203, P.R. China

E-mail: dihuangh@sina.com

Key words: chronic kidney failure, Tumor necrosis factor receptorassociated factor 5, apoptosis, berberine, nuclear factor- $\kappa \mathrm{B}$ by a glomerular filtration rate (GFR) persistently below $15 \mathrm{ml} / \mathrm{min} / 1.73 \mathrm{~m}^{2}$, and represents the end-stage of CKD requiring treatments, such as dialysis or transplantation. Cardiovascular disease and infection are two most frequent causes of death in patients with CRF (1). There are also other causes of death for such patients, which vary and these include cancer, cachexia, death attributable to social factors and other unknown causes (1-3). Kidney failure results in a decline in renal function, as evidenced by neurohumoral and metabolic abnormalities and the accumulation of damaging molecules, metabolic acidosis, electrolyte abnormalities and volume overload. Large observational databases have identified many hypothesis-generating risk factors for mortality in $\operatorname{CRF}(1,4)$. Despite some novel biomarkers which have been implicated in the risk of mortality (5), their effects on outcomes when used for therapeutic decisions have been insufficiently identified.

Berberine (BBR), the major pharmacological constituent of Coptis chinensis, is a type of isoquinoline alkaloid used as a therapeutic agent in the treatment of cancer, bacterial infections, diabetes, and cardiovascular and inflammatory diseases (6-8). Furthermore, accumulating evidence suggests that BBR can effectively inhibit cell proliferation and induce apoptosis, and that it has antioxidant properties $(9,10)$. Therefore, the present study aimed to examine the effects of BBR on the proliferation and apoptosis of mouse podocytes.

Tumor necrosis factor (TNF) receptor (TNFR)-associated factors (TRAFs) were originally identified as signaltransducing molecules for TNFR, but have also been linked to downstream signaling via other receptors, such as interleukin (IL)-1 receptor $(11,12)$. To date, 7 members of the TRAF family have been described. TRAF2, TRAF5 and TRAF6 have been demonstrated to mediate the activation of nuclear factor- $\kappa \mathrm{B}(\mathrm{NF}-\kappa \mathrm{B})$ by interacting with the mitogen-activated protein kinase (MAPK)/extracellular signal-regulated kinase (ERK1/2) pathway, the TNF- $\beta$-activated kinase, or the atypical protein, thus being implicated in the regulation of cell death and cellular responses to stress $(13,14)$. The TNF- $\alpha$-induced activation of NF- $\kappa \mathrm{B}$ has been shown to be severely inhibited in murine embryonic fibroblasts (MEFs) derived from TRAF5 knockout mice (15). Moreover, it has been reported that the TRAF5-induced activation of NF- $\kappa \mathrm{B}$ is involved in glioma cell migration and invasion (16). However, whether the TRAF5- 
induced $\mathrm{NF}-\kappa \mathrm{B}$ activation is involved in proliferation and apoptosis remains unknown.

In the present study, we examined the effects of BBR on mouse podocyte viability and apoptosis. We found that BBR prevented the induction of cell apoptosis induced by TRAF5 overexpression in mouse podocytes by suppressing NF- $\kappa$ B activation. Therefore, our results suggest that BBR plays an important role in the proliferation and apoptosis of mouse podocytes, and thus TRAF5 may be a potential therapeutic target in CKD.

\section{Materials and methods}

Bioinformatics analysis. The array expression data of TRAF5 for 13 patients with CKD and 12 healthy controls were downloaded from the NCBI Gene Expression Omnibus (GEO, http://www.ncbi.nlm.nih.gov/geo/) and are accessible through GEO Series accession no. GSE48944 (17), following the approval of this project by the consortium.

Patient samples. Serum samples were obtained from 30 patients with CRF and 30 healthy controls admitted to Shuguang Hospital, Shanghai, China. Ethical approval for the study was provided by the independent ethics committee, Shuguang Hospital of Shanghai University of Traditional Chinese Medicine. Written informed consent was obtained from all participants in this study. None of these patients had received radiotherapy or chemotherapy prior to obtaining the samples.

Cell culture. Mouse podocytes were obtained from the the BeNa Culture Collection (cat. no. BNCC100668; Beijing, China) and cultured in RMPI-1640 supplemented with $10 \%$ fetal bovine serum, $10 \%$ penicillin-streptomycin solution and $10 \mathrm{U} / \mathrm{ml}$ interferon- $\gamma($ IFN $-\gamma)$, and incubated in a humidified atmosphere at $33^{\circ} \mathrm{C}$ with $5 \% \mathrm{CO}_{2}$. Following culture for a period of time, the podocytes were cultured in the above-mentioned medium without $10 \mathrm{U} / \mathrm{ml}$ IFN- $\gamma$ and incubated in a humidified atmosphere at $37^{\circ} \mathrm{C}$ with $5 \% \mathrm{CO}_{2}$ for $10-14$ days.

Induction of TRAF5 overexpression in mouse podocytes. $\mathrm{pLV}$ IRES-eGFP, psPAX2 and pMD2G were obtained from Addgene (Cambridge, MA, USA). Commercial TRAF5 expression vectors were obtained from Genewiz Biotechnology (Suzhou, China). The TRAF5 expression sequence was cloned into the pLV-IRES-eGFP lentiviral vector. The blank pLV-IRES-eGFP lentiviral vector used as the negative control (NC). 293T cells (ATCC, Manassas, VA, USA) were seeded in $60 \mathrm{~mm}$ culture dishes, and after $24 \mathrm{~h}$, they were co-transfected with $2 \mu \mathrm{g}$ of the plasmid vector, $1 \mu \mathrm{g}$ pLV-IRES-eGFP-TRAF5/ pLV-IRES-eGFP, $0.1 \mu \mathrm{g}$ psPAX2 and $0.9 \mu \mathrm{g}$ pMD2G using Lipofectamine 2000 (Invitrogen, Thermo Fisher Scientific, Inc., Waltham, MA, USA) according to the manufacturer's instructions. The recombinant lentivirus was collected $48 \mathrm{~h}$ after transfection and used to infect the mouse podocytes.

Cell treatment. Following the induction of TRAF5 overexpression in mouse podocytes, the mouse podocytes were treated with various concentrations of BBR $(10,30$ and $90 \mu \mathrm{M}$; SigmaAldrich, St. Louis, MO, USA) and cell viability was measured by CCK- 8 assay to obtain the optimal concentration of BBR. To examine the effects of BBR and $\mathrm{NF}-\kappa \mathrm{B}$ on cell viability, apop- tosis and related protein expression, the mouse podocytes were treated with $30 \mu \mathrm{M}$ BBR or $25 \mu \mathrm{g} / \mathrm{ml}$ of the NF- $\kappa \mathrm{B}$ inhibitor, caffeic acid phenethyl ester (CAPE; Selleck, Shanghai, China) for $0,24,48$ and $72 \mathrm{~h}$ (for cell viability assay) or for $48 \mathrm{~h}$ (for cell apoptosis assay). To examine the effects of TNF- $\alpha$, IL-6 and LPS on cell viability, apoptosis and related protein expression, the mouse podocytes were treated with $100 \mathrm{ng} / \mathrm{ml} \mathrm{TNF-} \alpha$ (PeproTech, Rocky Hill, NJ, USA), 100 U/ml IL-6 (PeproTech) or $100 \mathrm{ng} / \mathrm{ml}$ LPS (Sigma-Aldrich) for 0, 24, 48 and $72 \mathrm{~h}$ (for cell viability assay) or for $48 \mathrm{~h}$ (for cell apoptosis assay) in the absence or presence of $30 \mu \mathrm{M}$ BBR treatment.

Reverse transcription and real-time PCR. Total RNA was extracted using TRIzol reagent (Invitrogen, Life Technologies, Carlsbad, CA, USA) as previously described (18). Complementary DNA was synthesized using a cDNA synthesis kit (Thermo Fisher Scientific Inc.). Real-time PCR was performed using a standard SYBR-Green PCR kit (Takara Biotechnology Co., Ltd., Dalian, China) and an ABI 7500 (Applied Biosystem Life Technologies, Foster City, CA, USA) thermal cycler. The primers used were as follows: TRAF5 forward, 5'-CACTCCGT GCTTCACAAC-3' and reverse, 5'-AAGGTGGTCCTGG AATCG-3'; glyceraldehyde 3-phosphate dehydrogenase (GAPDH) forward, 5'-ATCACTGCCACCCAGAAG-3' and reverse, 5'-TCCACGACGGACACATTG-3'. GAPDH was used an internal control for normalization. The gene expression was calculated using the $2^{-\Delta \Delta C q}$ method, as previously described (19).

Cell counting kit-8 (CCK-8) assay. Mouse podocytes infected with pLV-IRES-eGFP-TRAF5 $\left(3 \times 10^{3} /\right.$ well) were plated in 96-well plates. Following treatment as indicated for 0, 24, 48 and 72 h, 10\% of CCK-8 solution (Dojindo Molecular Technologies, Kumamoto, Japan) diluted in serum-free RMPI1640 was mixed in each well for a further $1 \mathrm{~h}$. The optical density $450 \mathrm{~nm}$ value in each well was determined by a microplate reader (SM600 Labsystem; Shanghai Utrao Medical Instrument Co., Ltd., Shanghai, China).

Apoptosis assay. Mouse podocytes infected with pLVIRES-eGFP-TRAF5 infection $\left(5 \times 10^{5} /\right.$ well) were plated in 6 -well plates. Follownig treatment as indicated for $48 \mathrm{~h}$, the mouse podocytes were collected and incubated with $195 \mu \mathrm{l}$ Annexin V-fluorescein isothiocyanate (FITC) and $5 \mu \mathrm{l}$ propidium iodide (PI) for $15 \mathrm{~min}$ in the dark at $4^{\circ} \mathrm{C}$, prior to analysis by flow cytometry (BD Biosciences, Franklin Lakes, NJ, USA)

Western blot analysis. Mouse podocytes were seeded at a density of $5 \times 10^{5}$ cells/well in 6-well plates, cultured overnight and then treated as indicated for 3 or $48 \mathrm{~h}$. Total proteins were isolated from the mouse podocytes and were subjected to $12 \%$ glyceraldehyde 3-phosphate dehydrogenase (SDS-PAGE) and electroblotted onto onto polyvinylidene fluoride membranes (Roche Diagnostics, Mannheim, Germany). The membranes were first incubated with rabbit polyclonal anti-Bax (1:300; Sc-493, Santa Cruz Biotechnology, Inc., Dallas, TX, USA), anti-caspase-3 (1:500; ab44976, Abcam, Cambridge, MA, USA) and anti-nephrin (1:500; ab58968, Abcam) antibodies; rabbit monoclonal anti-TRAF5 (1:1,000; ab137763, Abcam), anti-

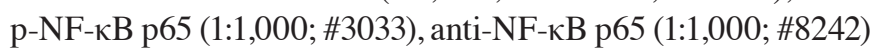
(both from Cell Signaling Technology, Danvers, MA, USA), anti- 
A

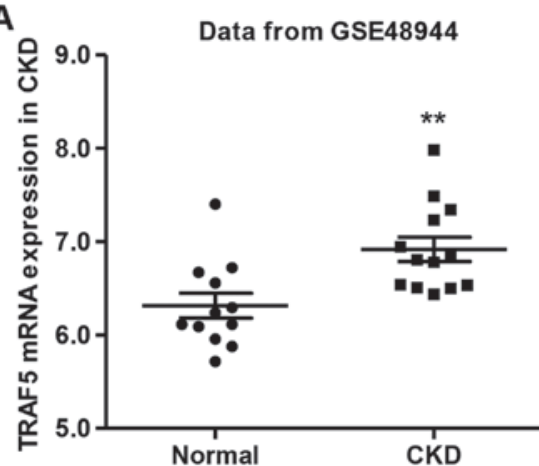

B

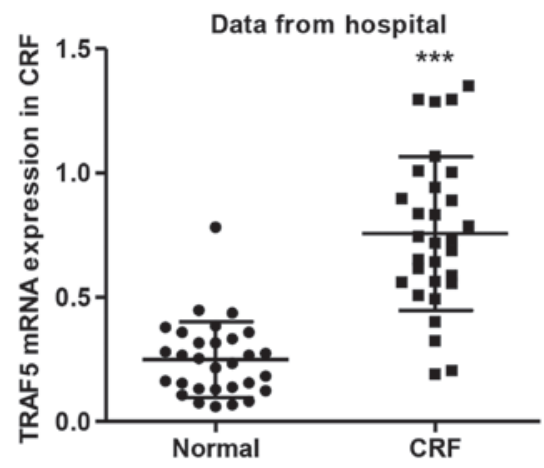

C

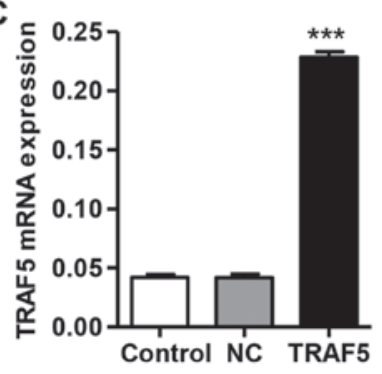

D

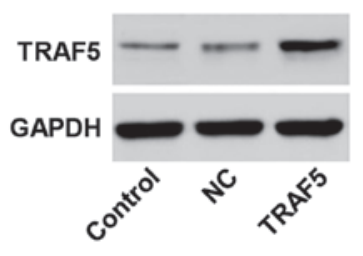

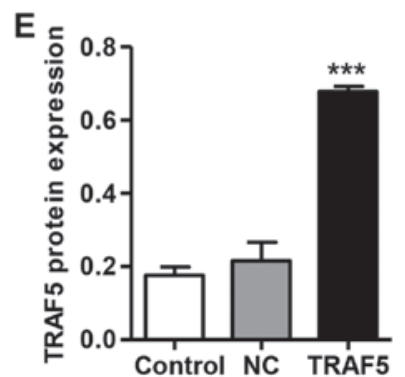

Figure 1. TNF receptor-associated factor 5 (TRAF5) levels in peripheral blood of patients with chronic kidney disease (CKD) and chronic renal failure (CRF). (A) The TRAF5 levels in peripheral blood of patients with CKD ( $\mathrm{n}=13$ ) and healthy controls ( $\mathrm{n}=12$ ) were measured by bioinformatics analysis. (B) The TRAF5 levels in peripheral blood of patients with CRF $(n=30)$ and healthy controls $(n=30)$ were measured by real-time PCR. Mouse podocytes were infected with pLV-IRES-eGFP-TRAF5 for $48 \mathrm{~h}$. (C) The mRNA level of TRAF5 was measured by real-time PCR. (D and E) The protein level of TRAF5 was measured by western blot analysis. ${ }^{* *} \mathrm{P}<0.01$ and ${ }^{* * *} \mathrm{P}<0.001$ vs. normal and negative control (NC).

podocin (1:10,000; ab181143, Abcam) and anti-GAPDH (1:1500; \#5174; Cell Signaling Technology) antibodies; and mouse monoclonal anti-Bcl-2 (1:400; ab117115, Abcam) antibody. The blots were then incubated with goat anti-mouse or anti-rabbit secondary antibody (1:1,000; A0208 and A0216, Beyotime Institute of Biotechnology, Haimen, China) and visualized using enhanced chemiluminescence (ECL; Thermo Fisher Scientific). GAPDH antibody was used as an internal control. The blotting bands were quantified with ImageJ software (National Institutes of Health, Bethesda, MD, USA).

Statistical analysis. All data are expressed as the means \pm SD and representative of experiments were carried out in triplicate analyzed with SPSS 19.0 software (SPSS Inc., Chicago, IL, USA). The unpaired, two-tailed Student's t-test and ANOVA followed by Tukey's post hoc test were used to analyze the significance of differences between groups. Differences were considered significant if the probability $(\mathrm{P})$-value was $<0.05$.

\section{Results}

TRAF5 levels in the peripheral blood of patients with CKD and $C R F$. In order to examine the role of TRAF5 in kidney disease, we first measured the levels of TRAF5 in the peripheral blood of patients with CKD $(n=13)$ and healthy controls $(n=12)$ using the data from the GSE48944 database. As shown in Fig. 1, TRAF5 mRNA expression was significantly increased in patients with CKD compared with the healthy controls. Furthermore, we also detected the levels of TRAF5 in the peripheral blood of patients with CRF $(n=30)$ and healthy controls $(n=30)$ from the
Shuguang Hospital database. As shown in Fig. 1B, similar to the data from the GSE48944 database, the mRNA expression of TRAF5 was significantly increased in patients with CRF (average, 0.756 and median, 0.726) compared with the healthy controls (average, 0.249 and median, 0.244).

To further examine the effects of TRAF5 on kidney function in vitro, mouse podocytes were infected with the TRAF5 overexpressionvector pLV-IRES-eGFP-TRAF5. As shown in Fig. 1C-E, the expression of TRAF5 was markedly increased at both the mRNA and protein level in the mouse podocytes infected with pLV-IRES-eGFP-TRAF5 compared with the controls. However, the podocytes infected wth the blank pLV-IRES-eGFP (NC) vector exhibited no changes in TRAF5 expression.

TRAF5 overexpression inhibits cell viability and induces the apoptosis of mouse podocytes. Following infection with pLV-IRES-eGFP-TRAF5, the mouse podocytes exhibited a significant decrease in cell viability in a time-dependent manner (Fig. 2A). After $72 \mathrm{~h}$ of incubation, the viability of the mouse podocytes infected with the TRAF5 overexpression vector was suppressed by $47.03 \pm 0.11 \%$ compared with the control group. However, the mouse podocytes infected with the blank pLV-IRES-eGFP (NC) vector exhibited no change in viability compared with the control. Furthermore, we also investigated the role of TRAF5 in the apoptosis of mouse podocytes. As shown in Fig. 2B and C, infection with the pLV-IRES-eGFP-TRAF5 vector increased the apoptosis $(35.9 \pm 0.8 \%)$ of mouse podocytes compared with the control group $(2.05 \pm 0.6 \%)$. However, infection of the mouse podocytes with the blank pLV-IRES-eGFP (NC) did not affect 

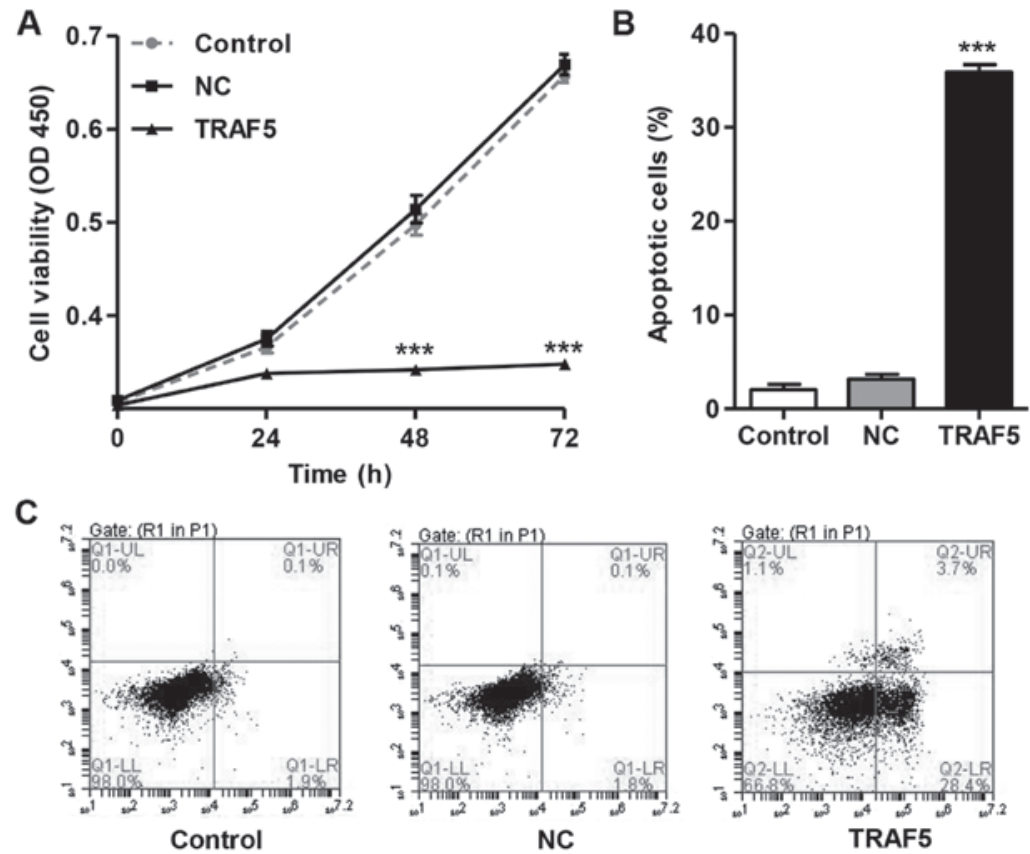

Figure 2. Effect of TNF receptor-associated factor 5 (TRAF5) overexpression on the viability and apoptosis of mouse podocytes. (A) The viability of mouse podocytes was measured by cell counting kit-8 (CCK-8) assay at the indicated time points. (B and C) The apoptosis of mouse podocytes was measured by flow cytometry. ${ }^{* * *} \mathrm{P}<0.001$ vs. negative control $(\mathrm{NC})$.
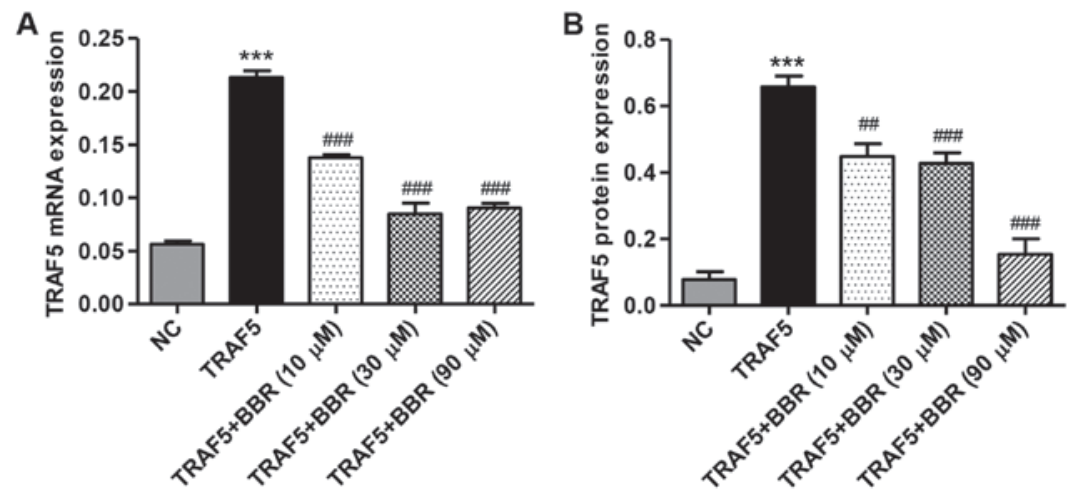

C

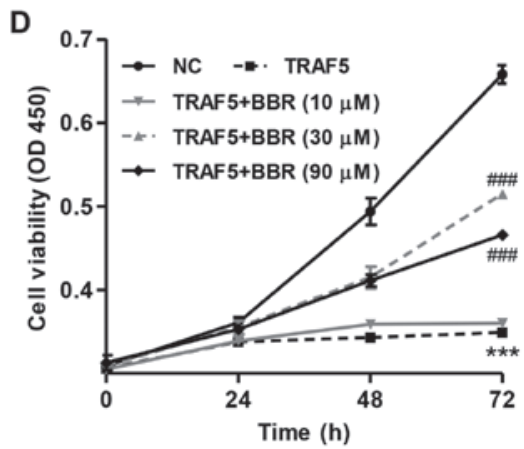

Figure 3. Berberine (BBR) suppresses the inhibition of cell viability and apoptosis induced by TNF receptor-associated factor 5 (TRAF5) overexpression. After the infection of mouse podocytes with pLV-IRES-eGFP-TRAF5, the cells were treated with BBR at the indicated concentrations. (A) The mRNA level of TRAF5 was measured by real-time PCR. (B and C) The protein level of TRAF5 was measured by western blot analysis. (D) The viability of mouse podocytes was measured by cell counting kit-8 (CCK-8) assay at the indicated time points. ${ }^{* * * *} \mathrm{P}<0.001$ vs. negative control (NC); ${ }^{\# \#} \mathrm{P}<0.01$ and ${ }^{\# \# \#} \mathrm{P}<0.001$ vs. TRAF5.

cell apoptosis compared with the control. These results indicate that TRAF5 overexpression is implicated in the inhibition of the viability and the apoptosis of mouse podocytes.

BBR suppresses TRAF5 expression and prevents the inhibition of cell viability induced by TRAF5. Considering the role of BBR in renal injury in experimental rats (20), and as the the mechanisms underlying the effects of BBR are not yet fully understood, we wished to determine whether BBR also possesses a exerts an effect on TRAF5 overexpression in podocytes. As shown in Fig. 3A, BBR (10, 30 and $90 \mu \mathrm{M})$ treatment significantly decreased the expression of TRAF5 at both the 
A

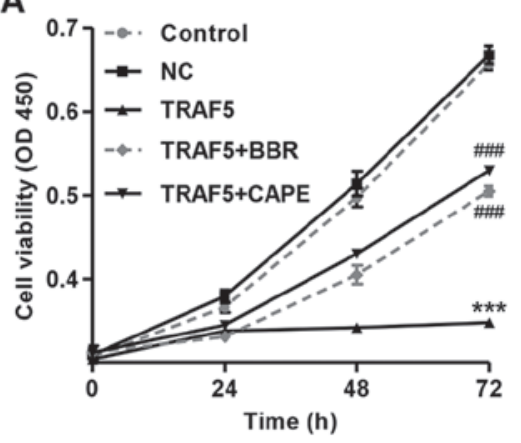

B

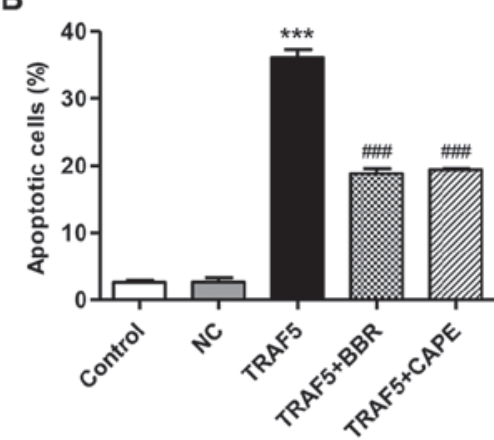

C

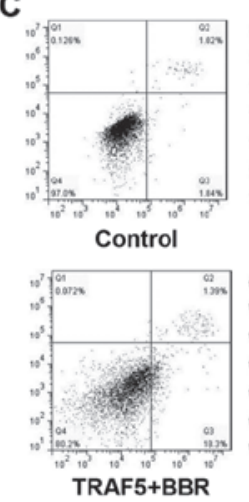

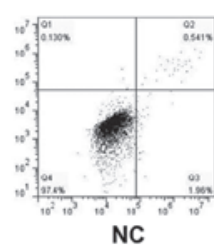

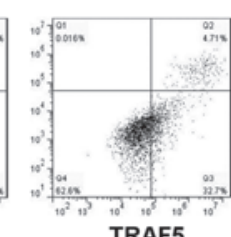

NC

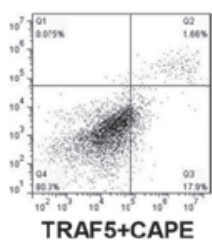

TRAF5
D

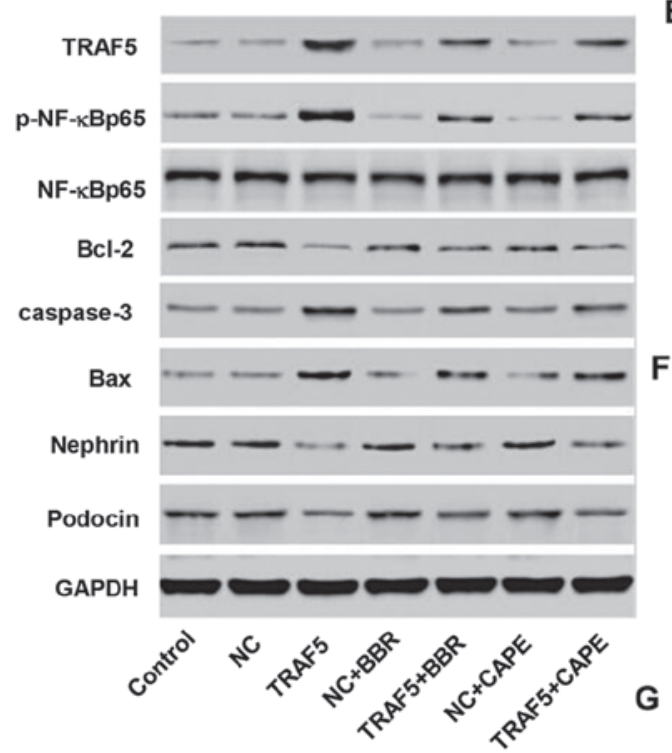

E

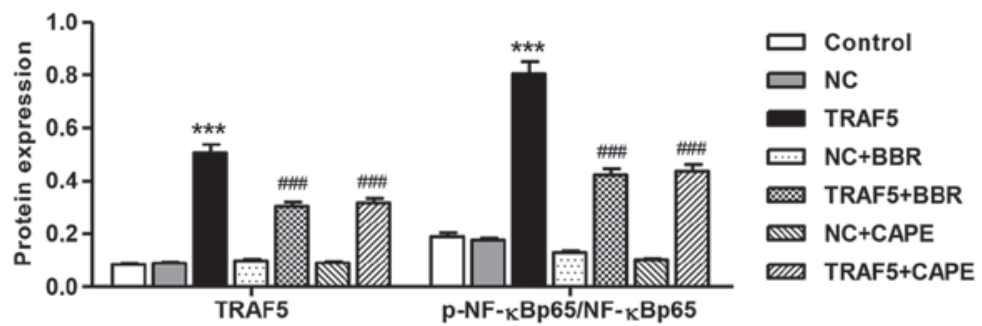

.
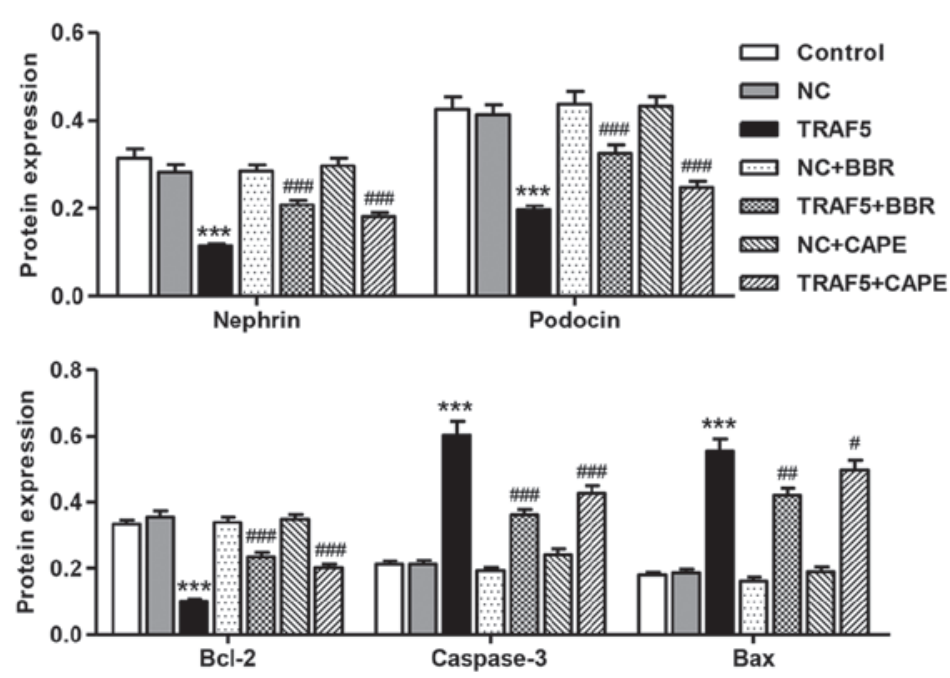

Figure 4. Berberine (BBR) suppresses nuclear factor- $\kappa \mathrm{B}(\mathrm{NF}-\kappa \mathrm{B})$ activation induced by TNF receptor-associated factor 5 (TRAF5) overexpression. (A) The viability of mouse podocytes was measured by cell counting kit-8 (CCK-8) assay at the indicated time points. (B and C) The apoptosis of mouse podocytes was

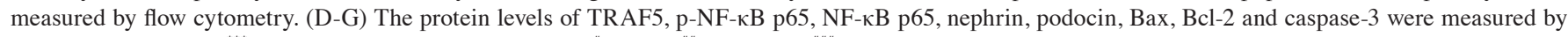
western blot analysis. ${ }^{* * *} \mathrm{P}<0.001$ vs. negative control $(\mathrm{NC}) ;{ }^{\#} \mathrm{P}<0.05,{ }^{\# \#} \mathrm{P}<0.01$ and ${ }^{\# \# \#} \mathrm{P}<0.001$ vs. TRAF5.

mRNA (Fig. 3A) and protein level (Fig. 3B and C) compared with the mouse podocytes infected with the TRAF5 overexpression vector and not treated with BBR. Compared with the podocytes infected with the TRAF5 overexpression vector and not treated with BBR, treatment of the mouse podocytes with various concentrations of BBR for 48 and $72 \mathrm{~h}$ increased cell viability in a time-dependent manner (Fig. 3D). After $72 \mathrm{~h}$ of incubation, the viability of the mouse podocytes treated with with BBR (30 and $90 \mu \mathrm{M}$ ) was increased by $47.5 \pm 2.6$ and $33.5 \pm 1.2 \%$, respectively compared with the podocytes infected with the TRAF5 overexpression vector and not treated with BBR. These findings suggest that the downregulation of TRAF5 is involved in the BBR-induced increase in podocyte viability.

$B B R$ suppresses the $N F-\kappa B$ activation induced by TRAF 5 overexpression. Following treatment with $30 \mu \mathrm{M}$ BBR or $25 \mu \mathrm{g}$ / $\mathrm{ml}$ CAPE for $72 \mathrm{~h}$, the viability of the mouse podocytes was significantly increased by $47.9 \pm 2.8$ and $52.3 \pm 3.5 \%$, respectively compared with the untreated podocytes infected with the TRAF5 overexpression vector (Fig. 4A). Treatment with BBR or CAPE treatment for $48 \mathrm{~h}$ also decreased the apoptosis of the mouse podocytes by $47.8 \pm 3.9$ and $46.1 \pm 2.5 \%$, respectively compared with the untreated podocytes infected with the TRAF5 overexpression vector (Fig. 4B and C).

To clarify the effects of TRAF5 on NF- $\kappa$ B p65 activation in vitro, western blot analysis was performed. As shown in Fig. 4D and $\mathrm{E}$, the ratio of p-NF- $\kappa \mathrm{B}$ p $65 / \mathrm{NF}-\kappa \mathrm{B}$ p65 was significantly decreased following treatment with $30 \mu \mathrm{M}$ BBR compared with the untreated podocytes infected with the TRAF5 overexpression vector. Similarly, CAPE $(25 \mu \mathrm{g} / \mathrm{ml})$, a potent and specific inhibitor of the activation of $N F-\kappa B$, mimicked the suppressive effects of BBR on TRAF5 overex- 
A

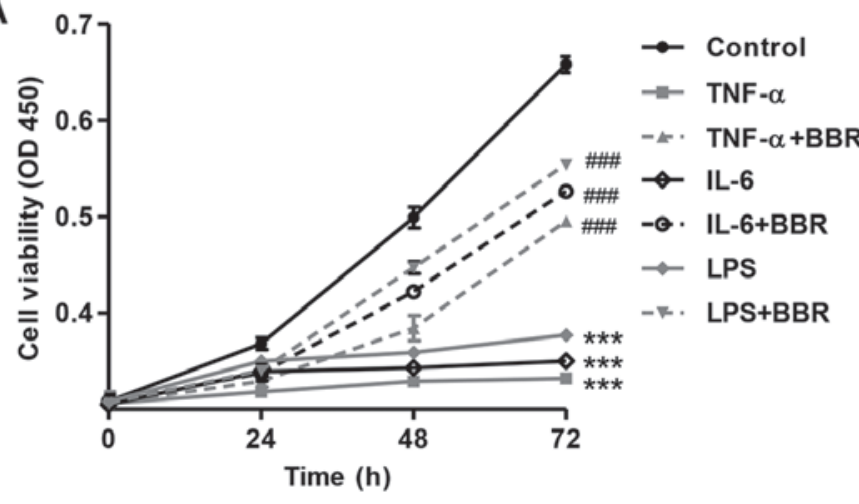

B

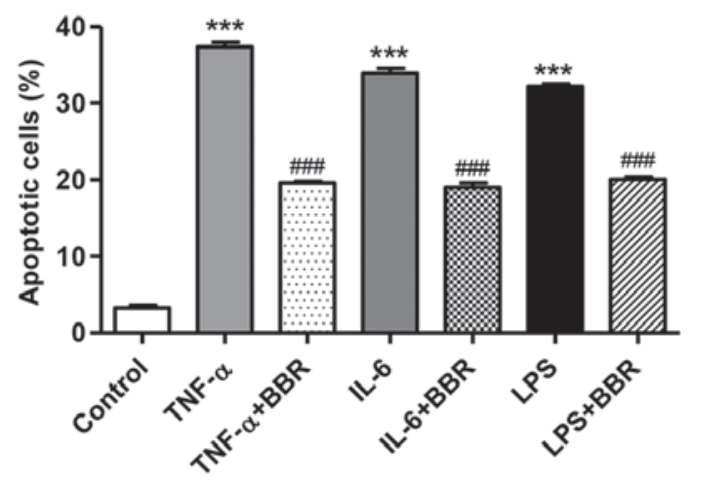

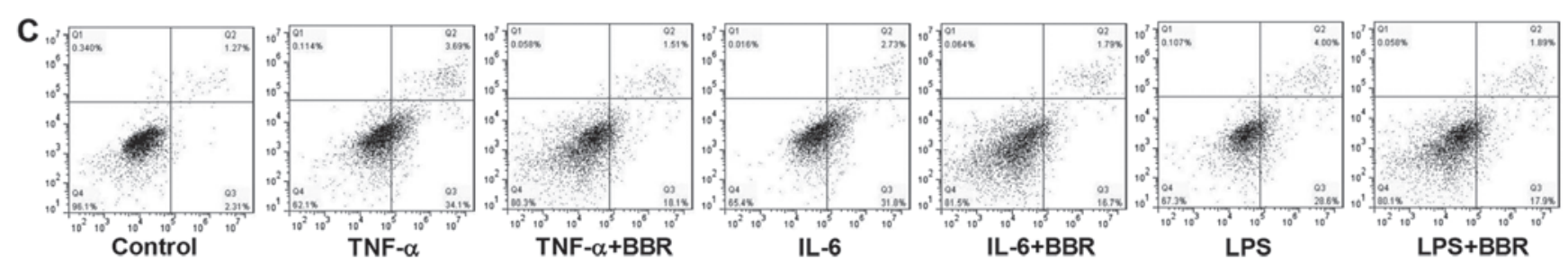

Figure 5. Berberine (BBR) suppresses the inhibition of cell viability and the apoptosis induced by tumor necrosis factor- $\alpha$ (TNF- $\alpha$ ), interleukin- 6 (IL-6) and LPS. (A) The viability of mouse podocytes was measured by cell counting kit- 8 (CCK-8) assay at the indicated time points. (B and C) The apoptosis of mouse podocytes was measured by flow cytometry. ${ }^{* * *} \mathrm{P}<0.001 \mathrm{vs}$. control; ${ }^{\# \# \#} \mathrm{P}<0.001$ vs. corresponding TNF- $\alpha$, IL- 6 and LPS treatment alone, respectively.

pression and NF- $\mathrm{\kappa B}$ p65 activation in mouse podocytes. These data demonstrate that the inactivation of NF- $\mathrm{KB}$ may contribute to the BBR-induced protective effects on mouse podocytes.

Effect of BBR and CAPE on protein expression induced by TRAF5 overexpression. Following the infection of the mouse podocytes with pLV-IRES-eGFP-TRAF5 for $48 \mathrm{~h}$, the expression of nephrin and podocin was significantly suppressed (Fig. 4D and F), while the Bax/Bcl-2 ratio and caspase- 3 levels were significantly increased compared with the control group (Fig. 4D and G). However, treatment with $30 \mu \mathrm{M}$ BBR or $25 \mu \mathrm{g} / \mathrm{ml}$ CAPE for $48 \mathrm{~h}$ suppressed the effects induced by TRAF5 overexpression on these protein expression levels in mouse podocytes (Fig. 4D-G).

$B B R$ prevents the inhibition of cell viability and the apoptosis induced by TNF- $\alpha, I L-6$ and LPS. Treatment with TNF- $\alpha(100 \mathrm{ng} / \mathrm{ml})$, IL-6 $(100 \mathrm{U} / \mathrm{ml})$ or LPS $(100 \mathrm{ng} / \mathrm{ml})$ for $72 \mathrm{~h}$ significantly decreased cell viability by $48.0 \pm 3.2$, $46.8 \pm 2.7$ and $45.1 \pm 2.9 \%$, respectively compared with the control group (Fig. 5A). However, treatment with $30 \mu \mathrm{M}$ BBR markedly prevented the inhibition of cell viability induced by TNF- $\alpha$, IL-6 or LPS in mouse podocytes. Moreover, treatment with $30 \mu \mathrm{M}$ BBR also suppressed the apoptosis induced by TNF- $\alpha$, IL- 6 or LPS by $47.6 \pm 2.6,43.9 \pm 3.3$ and $37.7 \pm 1.9 \%$, respectively compared with the cells exposed to TNF- $\alpha$, IL-6 or LPS (Fig. 5B and C). These data suggest that BBR inhibits TNF- $\alpha$-, IL- 6 - or LPS-induced cytotoxicity in mouse podocytes.

Effect of BBR on NF- $\kappa B$ activation and protein expressions induced by TNF- $\alpha, I L-6$ and LPS. As shown in Fig. 6, the ratio of p-NF- $\mathrm{kB}$ p $65 / \mathrm{NF}-\kappa \mathrm{B}$ p 65 and $\mathrm{Bax} / \mathrm{Bcl}-2$ and the expression levels of TRAF5 and caspase-3 were significantly increased by TNF- $\alpha$, IL- 6 or LPS treatment compared with the controls. However, treatment with $30 \mu \mathrm{M}$ BBR markedly suppressed the effects of TNF- $\alpha$, IL- 6 or LPS on NF- $\mathrm{BB}$ activation and on the expression levels of these proteins in mouse podocytes. These results indicate that TRAF5 downregulation is implicated in the protective effects of BBR against the effects of TNF- $\alpha$, IL-6 or LPS in mouse podocytes.

\section{Discussion}

This study reports the novel finding that TRAF5 expression was increased in the peripheral blood of patients with CKD and CRF. In vitro experiments revealed that TRAF5 overexpression inhibited the viability and induced the apoptosis of mouse podocytes. We further demonstrated that BBR inhibited the negative effects of TRAF5 overexpression by suppressing NF- $\mathrm{KB}$ activation in mouse podocytes. These data provide novel evidence (at least to the best of our knowledge) that BBR protects mouse podocytes from the suppressive effects of TRAF5 on viability and the promoting effects of TRAF5 on apoptosis via the NF- $\mathrm{BB}$ signaling pathway.

It has previously been reported that soluble TRAF5 levels are increased in plasma and peripheral blood mononuclear cells from patients with Crohn's disease and ulcerative colitis (21). Other studies have implicated TRAF5 in carcinogenesis attributable to increased levels in splenic marginal zone lymphoma (22) and Hodgkin-Reed-Sternberg cells (23). Intriguingly, the TRAF5/GAPDH mRNA ratios have been shown to be significantly decreased in the blood of patients with chronic and acute coronary heart disease, supporting the notion that TRAF5 represents a protective marker in atherosclerosis (24). However, the expression of TRAF5 in patients with CKD has not yet been elucidated. To examine the hypothesis that TRAF5 may also be 

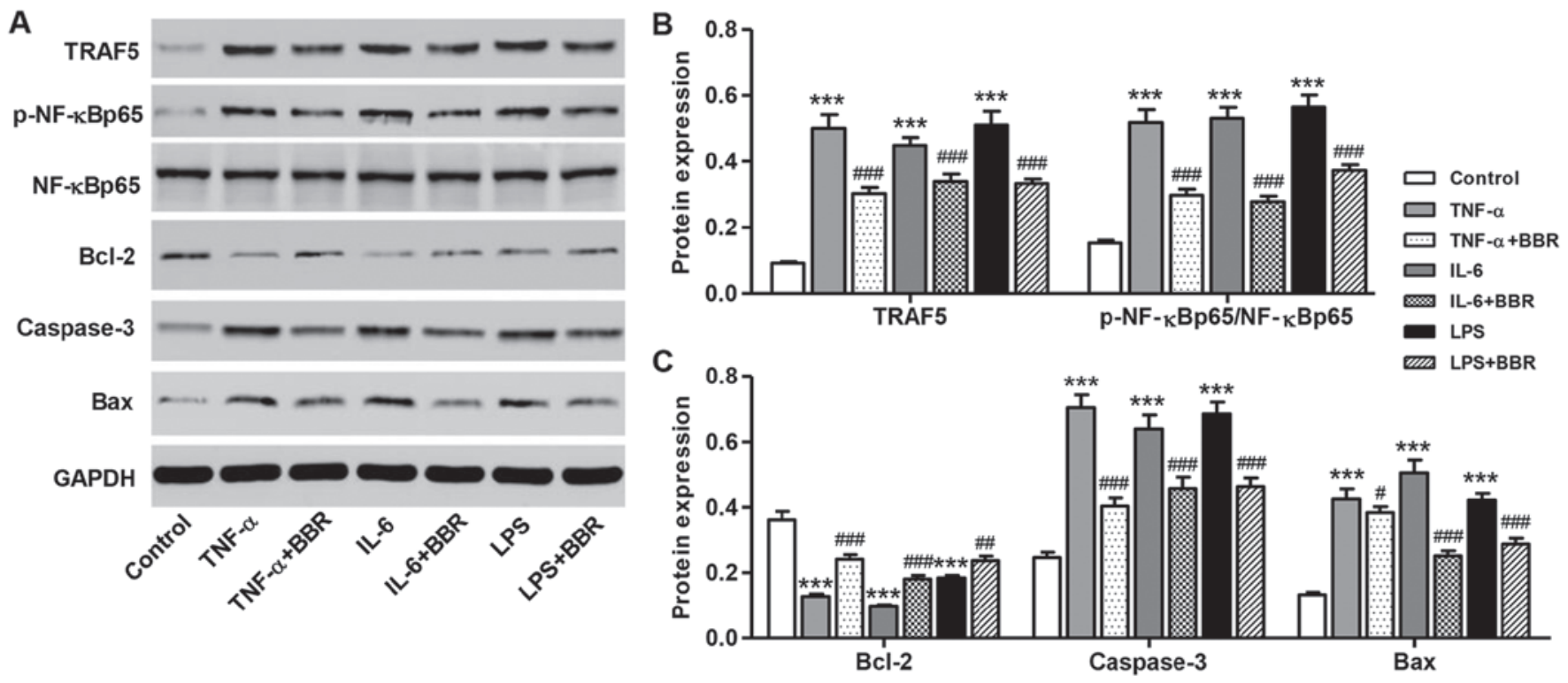

Figure 6. Effect of berberine (BBR) on nuclear factor- $\kappa \mathrm{B}(\mathrm{NF}-\kappa \mathrm{B})$ activation and protein expressions induced by tumor necrosis factor- $\alpha$ (TNF- $\alpha$ ), interleukin-6 (IL-6) and LPS. (A-C) The protein levels of TNF receptor-associated factor 5 (TRAF5), p-NF- $\kappa$ B p65 and NF- $\kappa$ B p65, Bax, Bcl-2 and caspase-3 were measured by western blot analysis. ${ }^{* * *} \mathrm{P}<0.001$ vs. control; ${ }^{\#} \mathrm{P}<0.05,{ }^{\# \#} \mathrm{P}<0.01$ and ${ }^{\# \# \#} \mathrm{P}<0.001$ vs. corresponding TNF- $\alpha$, IL-6 and LPS treatment alone, respectively.

associated with clinical disease, we performed bioinformatics analysis and a pilot clinical study in patients with CKD and CRF. This study revealed increased peripheral blood levels of TRAF5 in patients with CKD and CRF compared with the healthy controls. As reported previously, TRAF5 knockout had no effect on the viability of MEFs, while MEFs from double TRAF2 and TRAF5 knockout mice exhibited significantly decreased cell viability compared with wild-type and single TRAF2 or TRAF5 knockout mice, suggesting a critical role of TRAF2 rather than TRAF5 in protection from cell death (15). However, as demonstrated in another study, the elimination of TRAF5 expression significantly decreased the migration and invasion of the glioma cells, and although the underlying mechanisms were not elucidated, this may have bene due to the inhibition of cell viability and apoptosis induction (16). In the present study, to examine whether the podocyte apoptosis was mediated via TRAF5 overexpression, we infected mouse podocyte with a TRAF5 overexpression vector. We found that TRAF5 overexpression significantly induced mouse podocyte apoptosis.

TRAF5 was originally identified as an activator of interleukin-induced $\mathrm{NF}-\kappa \mathrm{B}$ signal transduction via its TRAF domain. NF- $\kappa \mathrm{B}$ comprises a family of transcription factors involved in the regulation of a wide variety of biological responses. It is generally accepted that $N F-\kappa B$ activation is responsible for apoptosis resistance $(25,26)$. However, there is evidence to support a pro-apoptotic role for $\mathrm{NF}-\kappa \mathrm{B}$. It has been speculated that NF- $\kappa \mathrm{B}$ may have a dual function, either as an inhibitor or an activator of apoptotic cell death, depending on the levels of RelA and c-Rel (27). In this study, TRAF5 overexpression in mouse podocytes led to NF- $\kappa \mathrm{B}$ activation, accompanied by an increased expression of caspase- 3 and an increased $\mathrm{Bax} / \mathrm{Bcl}-2$ ratio. Within cells, there is a machinery consisting of pro-apoptotic genes (Bax) that promote apoptosis and anti-apoptotic genes (Bcl-2) that function as suppressors of apoptosis, and the balance between these genes may be a determinant of apoptosis or cell survival. Despite extensive studies in either field, there is only limited information on the role of $\mathrm{Bcl}-2$ and $\mathrm{Bax}$ in CKD. In vivo, Bcl-2 and Bax proteins have not been detected in the kidneys during ischemia (28), whereas the overexpression of $\mathrm{Bcl}-2$ can suppress the apoptosis of renal tubule cells induced by hypoxia/reoxygenation (29). Moreover, an increase in the $\mathrm{Bax} / \mathrm{Bcl}-2$ ratio by hypoxia/reoxygenation or ischemia/reperfusion injury triggers Bax translocation to the mitochondria and cytochrome $c$ release to cytoplasm, and enhances caspase-3-mediated renal tubular apoptosis (30). Podocyte damage is a common feature in glomerular diseases with ultrastructural changes, with the reduced expression of components of the slit diaphragm, such as nephrin and podocin (31). The levels of nephrin and podocin have been shown to be significantly reduced in lupus nephritis, with these effects beginning from the earlier stages and becoming more pronounced at advanced histological forms (32). Our data reported that the expression of nephrin and podocin was significantly decreased in mouse podocytes overexpressing TRAF5.

$\mathrm{NF}-\kappa \mathrm{B}$ is activated by inflammatory cytokines and cellular stresses, including TNF, IL-1, LPS, UV or $\gamma$-irradiation. Thus far, TRAF5-deficient mice do not show substantial defects in TNF- $\alpha$-induced NF- $\kappa$ B activation, suggesting that TRAF5 plays a redundant role in TNF- $\alpha$-induced NF- $\kappa$ B activation (15). By contrast, TRAF5 acts downstream of ILs, including IL-1 $\beta$ and IL-6, and plays a key role in IL-1 $\beta /$ IL-6-mediated NF- $\kappa$ B activation during glioma migration and invasion (16). In the present study, we observed that the exposure of mouse podocytes to TNF- $\alpha$, IL-6 and LPS significant decreased cell viability and induced apoptosis. More importantly, the activation of $\mathrm{NF}-\kappa \mathrm{B}$ and the increased $\mathrm{Bax} / \mathrm{Bcl}-2$ ratio and caspase -3 expression were also observed in the podocytes exposed to TNF- $\alpha$, IL-6 and LPS. These findings suggest that TRAF5 induces podocyte 
injury, to a certain extent, through $\mathrm{NF}-\kappa \mathrm{B}$ activation induced by TNF- $\alpha$, IL-6 and LPS.

Emerging evidence has indicated that BBR has multiple beneficial effects in the treatment of diabetes and cardiovascular diseases $(33,34)$. However, the protective effects of BBR and its molecular mechanisms of action in CKD and chronic kidney injury remain to be determined. BBR has been shown to attenuate renal injury in diabetic C57BL/6 mice through the suppression of the SphK-S1P signaling pathway (35). Additionally, BBR has been shown to exert protective effects in the presence of high glucose related to the inhibition of glucose-induced apoptosis that in turn upregulates the expression of nephrin and podocin (36). In agreement with the previous study, our results demonstrated that BBR increases cell viability and inhibits apoptosis by upregulating the expression of nephrin and podocin, and downregulating the expression of caspase- 3 and the $\mathrm{Bax} / \mathrm{Bcl}-2$ ratio. The $\mathrm{NF}-\kappa \mathrm{B}$ inhibitor, CAPE, mimicked the protective effecs of BBR. Moreover, the NF- $\kappa \mathrm{B}$ activation induced by TRAF5 overexpression and exposure to TNF- $\alpha$, IL- 6 or LPS was significantly inhibited by $\mathrm{BBR}$, which is in line with the findings of a previous study that BBR ameliorates intrarenal inflammation and tubulointerstitial injury, at least in part, through the suppression of the $\mathrm{NF}-\kappa \mathrm{B}$ signaling pathway (37).

In conclusion, in this study, we demonstrate that TRAF5 is overexpressed in CRF and inhibits the viability and induces the apoptosis of mouse podocytes. Treatment of TRAF5overexpressing mouse podocytes with BBR suppressed the inhibition of viability, prevented apoptosis, decreased the Bax/ Bcl-2 and caspase-3 expression, and increased the expression of nephrin and podocin. Such effects appear to be mediated by the inhibition of $\mathrm{NF}-\kappa \mathrm{B}$ activation. Thus, BBR may play an important role in delaying the progression of chronic kidney injury by preserving renal structure and function in patients with CRF.

\section{Acknowledgements}

This study was supported by the Shanghai Three-Year Project of Traditional Chinese Medicine (nos. ZY3-JSFC-2-1026 and ZY3-CCCX-2-1003).

\section{References}

1. Ortiz A, Covic A, Fliser D, Fouque D, Goldsmith D, Kanbay M, Mallamaci F, Massy ZA, Rossignol P, Vanholder R, et al; Board of the EURECA-m Working Group of ERA-EDTA: Epidemiology, contributors to, and clinical trials of mortality risk in chronic kidney failure. Lancet 383: 1831-1843, 2014.

2. Stengel B: Chronic kidney disease and cancer: A troubling connection. J Nephrol 23: 253-262, 2010.

3. Hsu CY, Ordoñez JD, Chertow GM, Fan D, McCulloch CE and Go AS: The risk of acute renal failure in patients with chronic kidney disease. Kidney Int 74: 101-107, 2008.

4. Kalantar-Zadeh K, Block G, Humphreys MH and Kopple JD: Reverse epidemiology of cardiovascular risk factors in maintenance dialysis patients. Kidney Int 63: 793-808, 2003.

5. Ortiz A, Massy ZA, Fliser D, Lindholm B, Wiecek A, MartínezCastelao A, Covic A, Goldsmith D, Süleymanlar G, London GM, et al: Clinical usefulness of novel prognostic biomarkers in patients on hemodialysis. Nat Rev Nephrol 8: 141-150, 2011.

6. Shidfar F, Ebrahimi SS, Hosseini S, Heydari I, Shidfar S and Hajhassani G: The effects of Berberis vulgaris fruit extract on serum lipoproteins, apoB, apoA-I, homocysteine, glycemic control and total antioxidant capacity in type 2 diabetic patients. Iran J Pharm Res 11: 643-652, 2012.
7. Wang Y, Campbell T, Perry B, Beaurepaire C and Qin L: Hypoglycemic and insulin-sensitizing effects of berberine in high-fat diet- and streptozotocin-induced diabetic rats. Metabolism 60: 298-305, 2011.

8. Zha W, Liang G, Xiao J, Studer EJ, Hylemon PB, Pandak WM Jr, Wang G, Li X and Zhou H: Berberine inhibits HIV protease inhibitor-induced inflammatory response by modulating ER stress signaling pathways in murine macrophages. PLoS One 5: e9069, 2010.

9. Katiyar SK, Meeran SM, Katiyar N and Akhtar S: p53 cooperates berberine-induced growth inhibition and apoptosis of non-small cell human lung cancer cells in vitro and tumor xenograft growth in vivo. Mol Carcinog 48: 24-37, 2009.

10. Jiang Q, Liu P, Wu X, Liu W, Shen X, Lan T, Xu S, Peng J, Xie X and Huang H: Berberine attenuates lipopolysaccharide-induced extracelluar matrix accumulation and inflammation in rat mesangial cells: Involvement of NF- $\kappa \mathrm{B}$ signaling pathway. Mol Cell Endocrinol 331: 34-40, 2011.

11. Ichikawa H, Takada Y, Murakami A and Aggarwal BB: Identification of a novel blocker of I $\mathrm{B} \alpha \alpha$ kinase that enhances cellular apoptosis and inhibits cellular invasion through suppression of NF- $\kappa B$-regulated gene products. J Immunol 174: 7383-7392, 2005.

12. Jackson-Bernitsas DG, Ichikawa H, Takada Y, Myers JN, Lin XL, Darnay BG, Chaturvedi MM and Aggarwal BB: Evidence that TNF-TNFR1-TRADD-TRAF2-RIP-TAK1-IKK pathway mediates constitutive NF-kappaB activation and proliferation in human head and neck squamous cell carcinoma. Oncogene 26: 1385-1397, 2007.

13. Korchnak AC, Zhan Y, Aguilar MT and Chadee DN: Cytokineinduced activation of mixed lineage kinase 3 requires TRAF2 and TRAF6. Cell Signal 21: 1620-1625, 2009.

14. Khan KA, Abbas W, Varin A, Kumar A, Di Martino V, Dichamp I and Herbein G: HIV-1 Nef interacts with HCV Core, recruits TRAF2, TRAF5 and TRAF6, and stimulates HIV-1 replication in macrophages. J Innate Immun 5: 639-656, 2013.

15. Tada K, Okazaki T, Sakon S, Kobarai T, Kurosawa K, Yamaoka S, Hashimoto H, Mak TW, Yagita H, Okumura K, et al: Critical roles of TRAF2 and TRAF5 in tumor necrosis factorinduced NF- $\kappa$ B activation and protection from cell death. J Biol Chem 276: 36530-36534, 2001.

16. Tao T, Cheng C, Ji Y, Xu G, Zhang J, Zhang L and Shen A: Numbl inhibits glioma cell migration and invasion by suppressing TRAF5-mediated NF- $\mathrm{BB}$ activation. Mol Biol Cell 23: 2635-2644, 2012.

17. Ko YA, Mohtat D, Suzuki M, Park AS, Izquierdo MC, Han SY, Kang HM, Si H, Hostetter T, Pullman JM, et al: Cytosine methylation changes in enhancer regions of core pro-fibrotic genes characterize kidney fibrosis development. Genome Biol 14: R108, 2013.

18. Wang LN, Wang Y, Lu Y, Yin ZF, Zhang YH, Aslanidi GV, Srivastava A, Ling CQ and Ling C: Pristimerin enhances recombinant adeno-associated virus vector-mediated transgene expression in human cell lines in vitro and murine hepatocytes in vivo. J Integr Med 12: 20-34, 2014.

19. Saha SK, Roy S and Khuda-Bukhsh AR: Ultra-highly diluted plant extracts of Hydrastis canadensis and Marsdenia condurango induce epigenetic modifications and alter gene expression profiles in HeLa cells in vitro. J Integr Med 13: 400-411, 2015.

20. Wu D, Wen W, Qi CL, Zhao RX, Lü JH, Zhong CY and Chen YY: Ameliorative effect of berberine on renal damage in rats with diabetes induced by high-fat diet and streptozotocin. Phytomedicine 19: 712-718, 2012.

21. Shen J, Qiao YQ, Ran ZH and Wang TR: Upregulation and preactivation of TRAF3 and TRAF5 in inflammatory bowel disease. Int J Med Sci 10: 156-163, 2013.

22. Ruiz-Ballesteros E, Mollejo M, Rodriguez A, Camacho FI, Algara P, Martinez N, Pollán M, Sanchez-Aguilera A, Menarguez J, Campo E, et al: Splenic marginal zone lymphoma: Proposal of new diagnostic and prognostic markers identified after tissue and cDNA microarray analysis. Blood 106: 1831-1838, 2005.

23. Horie R, Watanabe T, Ito K, Morisita Y, Watanabe M, Ishida T, HigashiharaM,Kadin Mand Watanabe T:Cytoplasmicaggregation of TRAF2 and TRAF5 proteins in the Hodgkin-Reed-Sternberg cells. Am J Pathol 160: 1647-1654, 2002.

24. Missiou A, Rudolf P, Stachon P, Wolf D, Varo N, Aichele P, Colberg C, Hoppe N, Ernst S, Münkel C, et al: TRAF5 deficiency accelerates atherogenesis in mice by increasing inflammatory cell recruitment and foam cell formation. Circ Res 107: 757-766, 2010. 
25. Su VY-F and Yang K-Y: Mesenchymal stem cell-conditioned medium induces neutrophils apoptosis via inhibition of NF- $\mathrm{KB}$ pathway and increases endogenous pulmonary stem cells in endotoxin-induced acute lung injury. Eur Respir J 46 (Suppl 59): OA3520, 2015.

26. Arora R, Yates C, Gary BD, McClellan S, Tan M, Xi Y, Reed E, Piazza GA, Owen LB and Dean-Colomb W: Panepoxydone targets NF- $\mathrm{NB}$ and FOXM1 to inhibit proliferation, induce apoptosis and reverse epithelial to mesenchymal transition in breast cancer. PLoS One 9: e98370, 2014.

27. Chen F, Wang M, O'Connor JP, He M, Tripathi T and Harrison LE: Phosphorylation of PPARgamma via active ERK1/2 leads to its physical association with p65 and inhibition of NF-kappabeta. J Cell Biochem 90: 732-744, 2003.

28. Eschwege P1, Paradis V, Conti M, Loric S, Dumas F, Berteau P, Ahmed M, Droupy S, Charpentier B, Legrand A, et al: Bcl-2 and Bax expression on rat ischemic kidney. Transplant Proc 30: 2861-2862, 1998.

29. Saikumar P, Dong Z, Patel Y, Hall K, Hopfer U, Weinberg JM and Venkatachalam MA: Role of hypoxia-induced Bax translocation and cytochrome $c$ release in reoxygenation injury. Oncogene 17: 3401-3415, 1998.

30. Chien CT, Chang TC, Tsai CY, Shyue SK and Lai MK: Adenovirusmediated bcl-2 gene transfer inhibits renal ischemia/reperfusion induced tubular oxidative stress and apoptosis. Am J Transplant 5: 1194-1203, 2005

31. Ikezumi Y, Suzuki T,Karasawa T, Kawachi H,Nikolic-Paterson DJ and Uchiyama M: Activated macrophages downregulate podocyte nephrin and podocin expression via stress-activated protein kinases. Biochem Biophys Res Commun 376: 706-711, 2008.

32. Perysinaki GS, Moysiadis DK, Bertsias G, Giannopoulou I, Kyriacou K, Nakopoulou L, Boumpas DT and Daphnis E: Podocyte main slit diaphragm proteins, nephrin and podocin, are affected at early stages of lupus nephritis and correlate with disease histology. Lupus 20: 781-791, 2011.
33. Lee YS, Kim WS, Kim KH, Yoon MJ, Cho HJ, Shen Y, Ye JM, Lee CH, Oh WK, Kim CT, et al: Berberine, a natural plant product, activates AMP-activated protein kinase with beneficial metabolic effects in diabetic and insulin-resistant states. Diabetes 55: 2256-2264, 2006.

34. Derosa G, Maffioli P and Cicero AF: Berberine on metabolic and cardiovascular risk factors: An analysis from preclinical evidences to clinical trials. Expert Opin Biol Ther 12: 1113-1124, 2012.

35. Lan T, Shen X, Liu P, Liu W, Xu S, Xie X, Jiang Q, Li W and Huang $\mathrm{H}$ : Berberine ameliorates renal injury in diabetic C57BL/6 mice: Involvement of suppression of SphK-S1P signaling pathway. Arch Biochem Biophys 502: 112-120, 2010.

36. Chen L, Guo W, Zhang S, Lu W, Liao S and Li Y: Berberine prevents high glucose-induced cell viability inhibition and apoptosis in podocytes. Int J Clin Exp Med 9: 5942-5950, 2016.

37. Wan X, Chen X, Liu L, Zhao Y, Huang WJ, Zhang Q, Miao GG, Chen W, Xie HG and Cao CC: Berberine ameliorates chronic kidney injury caused by atherosclerotic renovascular disease through the suppression of NFאB signaling pathway in rats. PLoS One 8: e59794, 2013. 